\title{
P-986 Letter of Intent: Medium-Energy Antiproton Physics at Fermilab
}

\author{
David M. Asner \\ Carleton University, Ottowa, ON, Canada K1S 5B6 \\ Thomas J. Phillips \\ Duke University, Durham, N. Carolina 27708 USA
}

Giorgio Apollinari, Daniel R. Broemmelsiek, Charles N. Brown, David C. Christian, Paul Derwent, Keith Gollwitzer, Alan Hahn, Vaia Papadimitriou, Ray Stefanski, Steven Werkema, Herman B. White Fermilab, Batavia, IL 60510, USA

Wander Baldini, Giulio Stancari, Michelle Stancari INFN, Sezione di Ferrara, Ferrara, Italy

Gerald P. Jackson

Hbar Technologies, LLC, West Chicago, IL 60185, USA

Daniel M. Kaplan, ${ }^{*}$ Yagmur Torun, Christopher G. White Illinois Institute of Technology, Chicago, Illinois 60616, USA

HyangKyu Park

KyungPook National University, DaeGu, Korea

Todd K. Pedlar

Luther College, Decorah, IA 52101, USA

H. Richard Gustafson

University of Michigan, Ann Arbor, MI 48109, USA

Jerome Rosen

Northwestern University, Evanston, IL 60208, USA

Mitchell Wayne

Notre Dame University, Notre Dame, IN 46556, USA

Alak Chakravorty

St. Xavier University, Chicago, IL 60655, USA

E. Craig Dukes

University of Virginia, Charlottesville, Virginia 22903, USA

February 5, 2009 


\begin{abstract}
Fermilab has long had the world's most intense antiproton source. Despite this, the opportunities for medium-energy antiproton physics at Fermilab have been limited in the past and - with the antiproton source now exclusively dedicated to serving the needs of the Tevatron Collider - are currently nonexistent. The anticipated shutdown of the Tevatron in 2010 presents the opportunity for a world-leading medium-energy antiproton program. We summarize the current status of the Fermilab antiproton facility and review some physics topics for which the experiment we propose could make the world's best measurements. Among these, the ones with the clearest potential for high impact and visibility are in the area of charm mixing and $C P$ violation.

Continued running of the Antiproton Source following the shutdown of the Tevatron is thus one of the simplest ways that Fermilab can restore a degree of breadth to its future research program. The impact on the rest of the program will be minor. We request a small amount of effort over the coming months in order to assess these issues in more detail.
\end{abstract}

*Spokesperson. E-mail address: kaplan@iit.edu 


\section{Motivation}

The world's highest-energy and highest-intensity antiproton source is at Fermilab. Having previously supported medium-energy antiproton fixed-target experiments (including the charmonium experiments E760 and E835), it is now 100\% dedicated to providing luminosity for the Tevatron Collider. At CERN, the LEAR antiproton storage ring was decommissioned in $1996{ }^{1}$ its successor facility, the Antiproton Decelerator (AD), provides antiproton beams at momenta of 100 and $300 \mathrm{MeV} / c$, at intensities up to $\approx 2 \times 10^{7}$ per minute [1]. ${ }^{2}$ It is noteworthy that Germany has embarked on a $\approx$ billion-Euro upgrade plan for the GSIDarmstadt nuclear-physics laboratory that includes construction of 30 and $90 \mathrm{GeV}$ rapidcycling synchrotrons and low- and medium-energy antiproton storage rings [2].

A number of intriguing recent discoveries can be elucidated at a medium-energy antiproton facility, foremost among which is charm mixing [3]. The key question is whether there is new physics in charm mixing; the signature for this is $C P$ violation [4]. The search for new physics in $B$ and $K$ mixing and decay has so far come up empty. Thus it behooves us to look elsewhere as well. As pointed out by many authors, charm is an excellent venue for such investigation: It is the only up-type quark in which such effects are possible, and standard-model backgrounds to new physics in charm are suppressed by small CKM-matrix elements and the fact that the $b$ quark is the most massive one participating in loop diagrams [5]. We argue below that a charm experiment at the Fermilab Antiproton Source can be the world's most sensitive.

Other topics of interest include such states as the $X(3872)$ in the charmonium region [6], observed by several groups, as well as the investigation of possible new-physics signals observed in the HyperCP experiment at Femilab: evidence for $C P$ violation [7] and flavorchanging neutral currents in hyperon decay [8]. In addition, the $h_{c}$ mass and width, $\chi_{c}$ radiative-decay angular distributions, and $\eta_{c}^{\prime}(2 S)$ full and radiative widths, important parameters of the charmonium system that remain to be precisely determined, are well suited to the $\bar{p} p$ technique $[9,10]$. Table 1 lists energy and momentum thresholds for various processes that could be studied.

Charmed particles can be pair-produced in $\bar{p} p$ or $\bar{p} N$ collisions at and above the $\psi(3770)$ resonance. There is an enormous cross-section advantage relative to $e^{+} e^{-}$colliders: charm hadroproduction cross sections are typically $\mathcal{O}(10 \mu \mathrm{b})$, while $e^{+} e^{-}$cross sections are $\mathcal{O}(1 \mathrm{nb})$. Against this must be weighed the $e^{+} e^{-}$luminosity advantage, typically $\mathcal{O}\left(10^{2}\right)$. Charm hadroproduction at high energies comes with the advantage of longer decay distances, but the countervailing disadvantage of higher multiplicity $\left(\left\langle n_{c h}\right\rangle \sim 10\right)$ in the underlying event. We expect that the low charged-particle multiplicity $\left(\left\langle n_{c h}\right\rangle \approx 2\right)$ in $\bar{p} p$ collisions somewhat above open-charm threshold will enable charm samples with cleanliness comparable to that at the $B$ factories, with the application of only modest cuts, and hence, high efficiency. The competition for this program is a possible "super- $B$ factory." (See Sec. 3.1 below for further discussion.)

Fermilab Antiproton Accumulator experiments E760 and E835 made the world's most precise measurements of charmonium masses and widths $[9,10,11]$. The achieved precision $(\lesssim 100 \mathrm{keV})$ was made possible by the extraordinarily narrow energy spread of the stochas-

\footnotetext{
${ }^{1}$ LEAR was turned off in spite of its review committee's recommendation that it be allowed to complete its planned program of research; the rationale was to free up expert manpower for LHC work. The "ground rules" for the AD design accordingly required operability by as small a crew as possible.

${ }^{2}$ The AD accepts about $5 \times 10^{7}$ antiprotons per cycle at a momentum of $3.57 \mathrm{GeV} / c$, produced with $1.5 \times$ $10^{13}$ protons from the PS; the antiprotons are then cooled and decelerated for provision to the experiments.
} 
Table 1: Thresholds for some processes of interest and lab-frame $\bar{p}$ momentum for $\bar{p} p$ fixedtarget.

\begin{tabular}{|c|c|c|c|c|c|}
\hline \multirow[b]{2}{*}{ Hyperon pairs } & \multicolumn{2}{|c|}{ Threshold } & \multirow[b]{2}{*}{ "Charmonium" } & \multicolumn{2}{|c|}{ Threshold } \\
\hline & $\begin{array}{c}\sqrt{s} \\
(\mathrm{GeV})\end{array}$ & $\begin{array}{c}p_{\bar{p}} \\
(\mathrm{GeV} / c)\end{array}$ & & $\begin{array}{c}\sqrt{s} \\
(\mathrm{GeV})\end{array}$ & $\begin{array}{c}p_{\bar{p}} \\
(\mathrm{GeV} / c)\end{array}$ \\
\hline $\bar{p} p \rightarrow \bar{\Lambda} \Lambda$ & 2.231 & 1.437 & $\bar{p} p \rightarrow \eta_{c}$ & 2.980 & 3.678 \\
\hline $\bar{p} p \rightarrow \bar{\Sigma}^{-} \Sigma^{+}$ & 2.379 & 1.854 & $\bar{p} p \rightarrow \psi(3770)$ & 3.771 & 6.572 \\
\hline $\bar{p} p \rightarrow \bar{\Xi}^{+} \Xi^{-}$ & 2.642 & 2.620 & $\bar{p} p \rightarrow X(3872)$ & 3.871 & 6.991 \\
\hline $\bar{p} p \rightarrow \bar{\Omega}^{+} \Omega^{-}$ & 3.345 & 4.938 & $\bar{p} p \rightarrow X$ or $Y(3940)$ & 3.940 & 7.277 \\
\hline & & & $\bar{p} p \rightarrow Y(4260)$ & 4.260 & 8.685 \\
\hline
\end{tabular}

tically cooled antiproton beam and the absence of Fermi motion and negligible energy loss in the hydrogen cluster-jet target. The other key advantage of the antiproton-annihilation technique is its ability to produce charmonium states of all quantum numbers, in contrast to $e^{+} e^{-}$machines which produce primarily $1^{--}$states and the few states that couple directly to them, or (with relatively low statistics) states accessible in $B$ decay or in $2 \gamma$ production.

The E835 apparatus did not include a magnet, thus various cross sections needed to assess the performance of a new experiment remain unmeasured. However, they can be estimated with some degree of confidence. We propose to assemble, quickly and at modest cost, an "upgraded E835" spectrometer that includes a magnet. If these cross sections are of the expected magnitude, it should be possible with this apparatus to make the world's best measurements of charm mixing and $C P$ violation, as well as of the other effects mentioned above. (If desired, a follow-on experiment could then be designed for even greater sensitivity, taking full advantage of the capabilities of the Fermilab Antiproton Source.)

The PANDA experiment [12] at the Facility for Antiproton and Ion Research (FAIR) could measure these cross sections after PANDA and the FAIR facility at GSI are built. FAIR and PANDA have yet to start construction, and PANDA turn-on is scheduled for 2016. The yearly antiproton production goal of FAIR for the PANDA experiment is an order of magnitude less than what the Antiproton Source currently provides for the Tevatron program. It is likely that with some ingenuity and creativity, such a program is feasible at the world's best antiproton source despite current constraints at Fermilab.

\section{Capabilities of the Fermilab Antiproton Source}

The Antiproton Source now cools and accumulates antiprotons at a stacking rate of $\approx 20 \mathrm{~mA} / \mathrm{hr}$, making it the world's most intense operating or proposed facility (Table 2). Given the $474 \mathrm{~m}$ circumference of the Antiproton Accumulator, this represents a production rate of $\approx 2 \times 10^{11}$ antiprotons $/ \mathrm{hr}$. Given the $60 \mathrm{mb}$ annihilation cross section, it could thus support in principle a luminosity up to about $5 \times 10^{32} \mathrm{~cm}^{-2} \mathrm{~s}^{-1}$, with antiproton stacking $\approx 50 \%$ of the time and collisions during the remaining $\approx 50 \%$. However, we anticipate operating at $\lesssim 2 \times 10^{32} \mathrm{~cm}^{-2} \mathrm{~s}^{-1}$, which allows $\gtrsim 80 \%$ duty cycle, poses less of a challenge to detectors and triggers, and requires a smaller fraction of the protons from the Main Injector. Since this is an order of magnitude above the typical E835 luminosity of $2 \times 10^{31} \mathrm{~cm}^{-2} \mathrm{~s}^{-1}$ [9], it requires more intense stores than in E835, higher target density, or both of these. While 
Table 2: Antiproton intensities at existing and future facilities.

\begin{tabular}{lcccc}
\hline \hline \multirow{2}{*}{ Facility } & \multicolumn{2}{c}{ Stacking: } & Clock Hours & $\bar{p} /$ Yr \\
& Rate $\left(10^{10} / \mathrm{hr}\right)$ & Duty Factor & $/$ Yr & $\left(10^{13}\right)$ \\
\hline \hline CERN AD & & & 3800 & 0.4 \\
FNAL (Accumulator) & 20 & $15 \%$ & 5550 & 17 \\
FNAL (New Ring) & 20 & $90 \%$ & 5550 & 100 \\
GSI FAIR & 3.5 & $90 \%$ & 2780 & 9 \\
\hline \hline
\end{tabular}

the optimal choice is a matter for further study, it is already clear that the desired luminosity can be achieved, for example using the typical E835 store intensity along with a ten-times denser target (a denser gas jet or a hydrogen-pellet target [12], or a wire target in the beam halo [13]). Since the optimal target material and configuration depend on the physics topic to be studied, we are planning for multiple target options. Ideally these should be designed to be easily interchangeable between runs.

\section{Physics Goals}

Our main physics goal is charm mixing. To indicate the range of important questions that can all be addressed by a common apparatus, we also discuss a few other physics examples: studying the mysterious $X(3872)$ state, searching for hyperon $C P$ violation, and studying a recently discovered rare hyperon-decay mode that may be evidence for new physics.

\subsection{Charm Mixing and $C P$ Violation}

After a more than 20-year search, $D^{0}-\bar{D}^{0}$ mixing is now established at 9.8 standard deviations [3], thanks mainly to the $B$ factories. The level of mixing is consistent with the wide range of standard model (SM) predictions [4]; however, this does not preclude a significant and potentially detectable contribution from new physics [14]. Since new physics can affect the charge-2/3 ("up-type") quark sector differently than the down-type, it is important [15] to carry out such charm-meson studies - the only up-type system for which meson mixing can occur.

Particle physics faces two key mysteries: the origin of mass and the existence of multiple fermion generations. While the former may be resolved by the LHC, the latter appears to originate at higher mass scales, which can only be studied indirectly. Such effects as $C P$ violation, mixing, and flavor-changing neutral or lepton-number-violating currents may hold the key to physics at these new scales $[15,16,17,18]$. Because in the charm sector the SM contributions to these effects are small, these are areas in which charm studies can provide unique information. In contrast, in the $s$ - and $b$-quark sectors in which such studies are typically pursued, with the exception of certain rare and difficult-to-study modes, there are large SM contributions to mixing and $C P$ violation $[19,20]$. For new-physics searches, these constitute backgrounds.

Both direct and indirect $C P$ violation are possible in charm decay. The standard model predicts direct $C P$ violation only in singly Cabibbo-suppressed (SCS) charm decays, at the $\mathcal{O}\left(10^{-3}\right)$ level [21], arising from interference between tree-level and loop processes (Fig. 1). The observation of larger $C P$ asymmetries than this would be unambiguous evidence for new physics; so too would nonzero $C P$ asymmetries in almost any Cabibbo-favored $(\mathrm{CF})$ or dou- 
a)

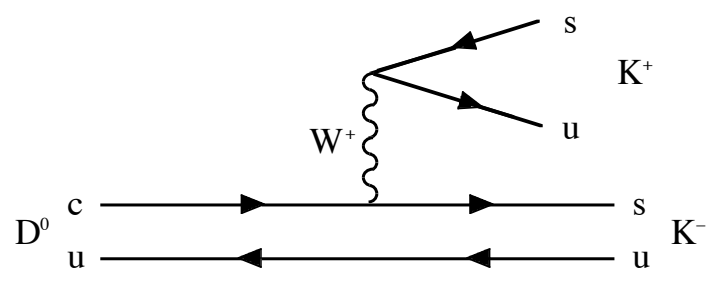

b)

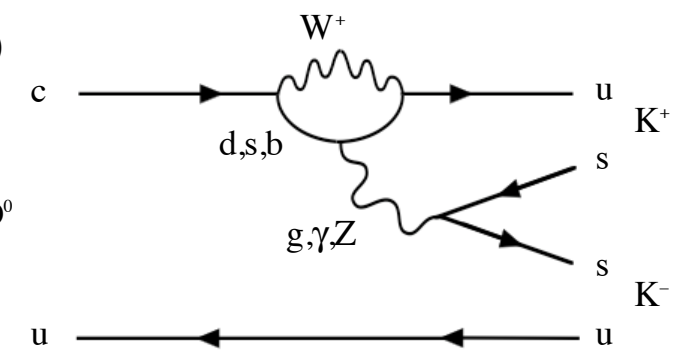

Figure 1: Example of singly Cabibbo-suppressed $D^{0}$ decay that can proceed through both a) tree and b) penguin diagrams.

bly Cabibbo-suppressed (DCS) charm decays, for which interfering SM penguin diagrams are absent. ${ }^{3}$

The experimental signature for direct $C P$ violation is a difference in partial decay rates between particle and antiparticle:

$$
A \equiv \frac{\Gamma(D \rightarrow f)-\Gamma(\bar{D} \rightarrow \bar{f})}{\Gamma(D \rightarrow f)+\Gamma(\bar{D} \rightarrow \bar{f})} \neq 0
$$

where $f$ and $\bar{f}$ are $C P$-conjugate final states. For $C P$-eigenstate final states, $f=\bar{f}$, the two processes of Eq. 1 are distinguished by initial-state tagging (e.g., $D^{* \pm} \rightarrow{ }^{(} \bar{D}^{0} \pi^{ \pm}$), while for $f \neq \bar{f}$, the final states are self-tagging. (Any production or efficiency asymmetries between particle and antiparticle can be normalized using a CF mode.)

\subsection{Charm Sensitivity Estimate}

The $\bar{p} p$ annihilation cross section to open charm is expected to be substantial; for example, a recent estimate (expected good to a factor of 3 [22]) based on $K^{*} \bar{K}$ measurements gives $\sigma\left(\bar{p} p \rightarrow D^{* 0} \bar{D}^{0}\right) \approx 1.3 \mu \mathrm{b}$ at $\sqrt{s}=4.2 \mathrm{GeV}$ [23] (Fig. 2). At $\mathcal{L}=2 \times 10^{32} \mathrm{~cm}^{-2} \mathrm{~s}^{-1}$, this represents some $5 \times 10^{9}$ events per year, substantially exceeding each year the total sample $\left(\approx 10^{9}\right.$ events) available at the $B$ factories. Since there will also be $D^{* \pm} D^{\mp}, D^{*} \bar{D}^{*}$, and $D \bar{D}$ events, the total charm sample will be even larger ${ }^{4}$ with the use of a target nucleus heavier than hydrogen, the charm-production $A$-dependence [26] could further enhance statistics by a factor of a few, resulting in reconstructed event samples of $\mathcal{O}\left(10^{9}\right) /$ year. Such a target could also localize primary interactions to an $\mathcal{O}(\mu \mathrm{m})$-sized region, allowing the $D$-meson decay distance to be cleanly determined, as required both for background suppression and for time-dependent mixing and $C P$-violation studies.

Initial simulations show that $\approx 50 \%$ acceptance can be achieved for $\bar{p} p \rightarrow D^{*} \bar{D}$ events, with the $D$ 's decaying to typical low-multiplicity final states. Further simulation studies are in progress. Evaluation of backgrounds requires either a reliable model for minimum-bias interactions or actual $\bar{p} p$ data at the appropriate energy. Extrapolations based on MIPP data at somewhat higher energies are underway. Results from these studies will be reported as they become available.

\footnotetext{
${ }^{3}$ The exception is SM asymmetries of $\approx 3.3 \times 10^{-3}\left(=2 \operatorname{Re}\left(\epsilon_{K}\right)\right)$ due to $K^{0}$ mixing in such modes as $D^{+} \rightarrow K_{S} \pi^{+}$and $K_{S} \ell \nu$ [24].

${ }^{4}$ While one might naively expect these states to be populated according to spin statistics [22], this is not the case for $K^{*} \bar{K}$ and $K^{*} \bar{K}^{*}$ production, which are comparable [25].
} 


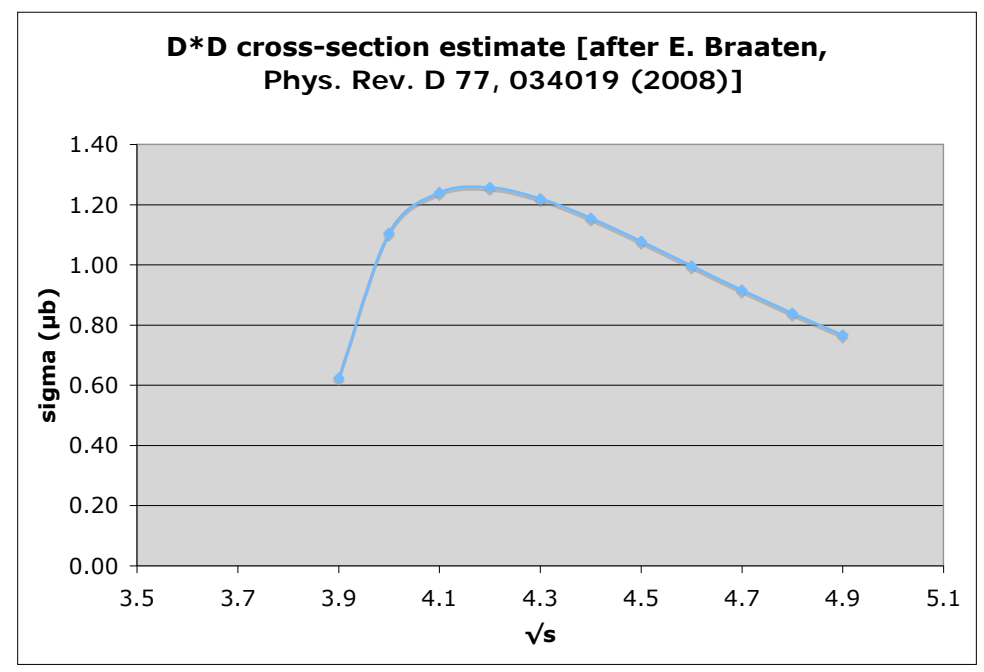

Figure 2: Estimated cross section for $\bar{p} p \rightarrow D^{* 0} \bar{D}^{0}$ (based on Ref. [23]).

Medium-energy $\bar{p} N$ annihilation may thus be the optimal way to study charm mixing and search for possible new-physics contributions via the clean signature [27] of charm $C P$ violation (CPV). The Fermilab Antiproton Source, with $8 \mathrm{GeV}$ design kinetic energy (maximum $\sqrt{s}=4.3 \mathrm{GeV}$ ), is ideally suited for this purpose. With an effective $\mathcal{O}(10 \mu \mathrm{b}$ ) total charm cross section, with much lower background-event multiplicities than at high energy, and with a possibly higher tagging efficiency than at the $B$ factories, the Fermilab Antiproton Source may well be a gold mine for new-physics searches and studies. Can Fermilab (and US HEP) afford to pass this up?

\section{$3.3 \quad X(3872)$}

The $X(3872)$ was discovered in 2003 by the Belle Collaboration [29] via the decay sequence $B^{ \pm} \rightarrow K^{ \pm} X(3872), X(3872) \rightarrow \pi^{+} \pi^{-} J / \psi$; its existence was quickly confirmed by CDF [30], $\mathrm{D} \varnothing$ [31], and BaBar [32]. It has now been seen in the $\gamma J / \psi$ [33], $\gamma \psi^{\prime}[34], \pi^{+} \pi^{-} \pi^{0} J / \psi[35]$, and $D^{0} \bar{D}^{0} \pi^{0}[36]$ modes as well (Table 3 ). This state does not appear to fit within the charmonium spectrum. Although well above open-charm threshold, its observed width is $<2.3 \mathrm{MeV}$ at $90 \%$ C.L. [28], implying that decays to $D \bar{D}$ are forbidden and suggesting unnatural parity, $P=(-1)^{J+1}[37]$. It is a poor candidate for the $\psi_{2}\left(1^{3} D_{2}\right)$ or $\psi_{3}\left(1^{3} D_{3}\right)$ charmonium levels $[6,35,37]$ due to the nonobservation of radiative transitions to $\chi_{c}$. The observation of $X(3872) \rightarrow \gamma J / \psi$ implies positive $C$-parity, and additional observations essentially rule out all possibilities other than $J^{P C}=1^{++}[38,39]$. With those quantum numbers, the only available charmonium assignment is $\chi_{c 1}^{\prime}\left(2^{3} P_{1}\right)$; however, this is highly disfavored [6, 37] by the observed rate of $X(3872) \rightarrow \gamma J / \psi$. In addition, the plausible identification of $Z(3930)$ as the $\chi_{c 2}^{\prime}\left(2^{3} P_{2}\right)$ level suggests [6] that the $2^{3} P_{1}$ should lie some $49 \mathrm{MeV} / c^{2}$ higher in mass than the observed $m_{X}=3872.2 \pm 0.8 \mathrm{MeV} / c^{2}[28]$.

Inspired by the coincidence of the $X(3872)$ mass and the $D^{0} \bar{D}^{* 0}$ threshold, a number of ingenious solutions to this puzzle have been proposed, including an $S$-wave cusp [40] or a tetraquark state [41]. Perhaps the most intriguing possibility is that the $X(3872)$ represents the first clear-cut observation of a meson-antimeson molecule: specifically, a bound state of 
Table 3: Experimental observations of $X(3872)$.

\begin{tabular}{lcccc}
\hline \hline Experiment & Year & Mode & Events & Ref. \\
\hline \hline Belle, BaBar & 2003,2004 & $\pi^{+} \pi^{-} J / \psi$ & $35.7 \pm 6.8,25.4 \pm 8.7$ & {$[29,32]$} \\
CDF, DØ & 2004 & $\pi^{+} \pi^{-} J / \psi$ & $730 \pm 90,522 \pm 100$ & {$[30,31]$} \\
Belle & 2004 & $\omega\left(\pi^{+} \pi^{-} \pi^{0}\right) J / \psi$ & $10.6 \pm 3.6$ & {$[35]$} \\
Belle & 2005 & $\gamma J / \psi$ & $13.6 \pm 4.4$ & {$[33]$} \\
Belle & 2006 & $D^{0} \bar{D}^{* 0}$ & $23.4 \pm 5.6$ & {$[36]$} \\
BaBar & 2008 & $\gamma \psi^{\prime}$ & & {$[34]$} \\
\hline \hline
\end{tabular}

$D^{0} \bar{D}^{* 0}+D^{* 0} \bar{D}^{0}[42] .{ }^{5}$ A key measurement is then the precise mass difference between the $X$ and that threshold; if the molecule interpretation is correct, it should be very slightly negative, in accord with the small molecular binding energy [39]:

$$
0<E_{X}=\left(m_{D^{0}}+m_{D^{* 0}}-m_{X}\right) c^{2} \ll 10 \mathrm{MeV} .
$$

A direct and precise measurement of the width, which $\bar{p} p$ can provide $[9,10,11]$, is also highly desirable.

With the current world-average values [28] $m_{D^{0}}=1864.84 \pm 0.17 \mathrm{MeV} / c^{2}$ and $m_{D^{* 0}}-$ $m_{D^{0}}=142.12 \pm 0.07 \mathrm{MeV} / c^{2}$, we have $E_{X}=-0.4 \pm 0.8 \mathrm{MeV} / c^{2}$. By taking advantage of the small momentum spread and precise momentum-calibration capability of the Antiproton Accumulator, a $\bar{p} p \rightarrow X(3872)$ formation experiment can make extremely precise $\left(\lesssim 100 \mathrm{keV} / c^{2}\right)$ measurements of $m_{X}$, and directly measure $\Gamma_{X}$ to a similar precision, by scanning across the resonance. Additional important measurements include $\mathcal{B}\left[X(3872) \rightarrow \pi^{0} \pi^{0} J / \psi\right]$ to confirm the $C$-parity assignment [43].

\subsection{1 $X(3872)$ sensitivity estimate}

The production cross section of $X(3872)$ in $\bar{p} p$ annihilation has not been measured, but it has been estimated to be similar in magnitude to that of the $\chi_{c}$ states $[44,23]$. In E760, the $\chi_{c 1}$ and $\chi_{c 2}$ were detected in $\chi_{c} \rightarrow \gamma J / \psi$ (branching ratios of $36 \%$ and $20 \%$, respectively [28]) with acceptance times efficiency of $44 \pm 2 \%$, giving about 500 observed events each for an integrated luminosity of $1 \mathrm{pb}^{-1}$ taken at each resonance [45]. At $2 \times 10^{32} \mathrm{~cm}^{-2} \mathrm{~s}^{-1}$, the lower limit $\mathcal{B}\left[X(3872) \rightarrow \pi^{+} \pi^{-} J / \psi\right]>0.042$ at $90 \%$ C.L. [46] implies $\gtrsim 8 \times 10^{3}$ events in that mode per nominal month $\left(1.0 \times 10^{6} \mathrm{~s}\right)$ of running. By way of comparison, Table 3 shows current sample sizes, which are likely to increase by not much more than an order of magnitude as these experiments complete during the current decade. ${ }^{6}$ (Although CDF and $\mathrm{D} \varnothing$ could amass samples of order $10^{4} X(3872)$ decays, the large backgrounds in the $\mathrm{CDF}$ and $\mathrm{D} \varnothing$ observations, reflected in the uncertainties on the numbers of events listed in Table 3, limit their incisiveness.)

Given the uncertainties in the cross section and branching ratios, the above may well be an under- or overestimate of the $\bar{p} p$ formation and observation rates, perhaps by as much as

\footnotetext{
${ }^{5}$ Alternatively, the mass coincidence may be merely accidental, and the $X(3872)$ a $c \bar{c}$-gluon hybrid state; however, the mass and $1^{++}$quantum numbers make it a poor match to lattice-QCD predictions for such states [6].

${ }^{6}$ The $\bar{p} p \rightarrow X(3872)$ sensitivity will be competitive even with that of the proposed SuperKEKB [47] upgrade, should that project go forward.
} 
an order of magnitude. Nevertheless, it appears that a new experiment at the Antiproton Accumulator could obtain the world's largest clean samples of $X(3872)$, in perhaps as little as a month of running. The high statistics, event cleanliness, and unique precision available in the $\bar{p} p$ formation technique could enable the world's smallest systematics. Such an experiment could thus provide a definitive test of the nature of the $X(3872)$.

\subsection{Hyperon $C P$ violation}

In addition to the well-known $C P$-violation effects in kaon and $B$-meson mixing and decay [28], the standard model predicts slight $C P$ asymmetries in decays of hyperons $[48,49,50]$. In the kaon and beauty systems, such effects appear to be dominated by standard model processes. It thus behooves us to study other systems (such as charm and hyperons) as well, in which the signatures of new physics might stand out more sharply. Although both hyperon and kaon decay occur due to unstable $s$ quarks, theoretical analysis has shown that hyperon $C P$ asymmetries are in fact complementary to those in $K$ decays in their sensitivity to new physics (see e.g. $[50,51]$ ).

Hyperon $C P$ violation would of course be of the direct type since hyperon mixing would violate conservation of baryon number. The hyperon $C P$ asymmetries considered most accessible have involved comparison of the angular distributions of the decay products of polarized hyperons with those of the corresponding antihyperons [49]; however, partial-rate asymmetries are also expected [52, 53] and (as discussed below) may be detectable. More than one hyperon $C P$ asymmetry may be measurable in medium-energy $\bar{p} p$ annihilation to hyperon-antihyperon pairs. To be competitive with previous $\Xi$ and $\Lambda$ angular-distribution asymmetry measurements would require higher luminosity $\left(\sim 10^{33}\right)$ than is likely to be available, as well as a very substantial upgrade relative to the E835 apparatus. While summarizing the state of hyperon $C P$ asymmetries generally, for the purposes of this LoI we therefore emphasize in particular the $\Omega^{-} / \bar{\Omega}^{+}$partial-rate asymmetry, for which there is no previous measurement.

By angular-momentum conservation, in the decay of a spin- $1 / 2$ hyperon to a spin- $1 / 2$ baryon plus a pion, the final state must be either $S$-wave or $P$-wave. ${ }^{7}$ As is well known, the interference term between the $S$ - and $P$-wave decay amplitudes gives rise to parity violation, described by Lee and Yang [54] in terms of two independent parameters $\alpha$ and $\beta: \alpha$ is proportional to the real and $\beta$ to the imaginary part of this interference term. $C P$ violation can be sought as a difference in $|\alpha|$ or $|\beta|$ between a hyperon decay and its $C P$ conjugate antihyperon decay or as a particle-antiparticle difference in the partial widths for such decays $[49,55]$. For a precision angular-distribution asymmetry measurement, it is necessary to know the relative polarizations of the initial hyperons and antihyperons to high precision.

\subsubsection{Angular-distribution asymmetries}

Table 4 summarizes the experimental situation. The first three experiments cited studied $\Lambda$ decay only $[56,57,58]$, setting limits on the $C P$-asymmetry parameter $[49,55]$

$$
A_{\Lambda} \equiv \frac{\alpha_{\Lambda}+\bar{\alpha}_{\Lambda}}{\alpha_{\Lambda}-\bar{\alpha}_{\Lambda}}
$$

\footnotetext{
${ }^{7}$ A similar argument holds for a spin-3/2 hyperon, but involving $P$ and $D$ waves.
} 
where $\alpha_{\Lambda}\left(\bar{\alpha}_{\Lambda}\right)$ characterizes the $\Lambda(\bar{\Lambda})$ decay to (anti)proton plus charged pion. If $C P$ is a good symmetry in hyperon decay, $\alpha_{\Lambda}=-\bar{\alpha}_{\Lambda}$.

The need for precision knowledge of the initial-hyperon polarization can be finessed by using the cascade decay of charged- $\Xi$ hyperons to produce polarized $\Lambda$ 's, in whose subsequent decay the slope of the (anti)proton angular distribution in the "helicity" frame measures the product of $\alpha_{\Xi}$ and $\alpha_{\Lambda}$. This approach has been taken by Fermilab E756 [59] and CLEO [60]. If $C P$ is a good symmetry in hyperon decay this product should be identical for $\Xi^{-}$and $\bar{\Xi}^{+}$events. The $C P$-asymmetry parameter measured is thus

$$
A_{\Xi \Lambda} \equiv \frac{\alpha_{\Xi} \alpha_{\Lambda}-\bar{\alpha}_{\Xi} \bar{\alpha}_{\Lambda}}{\alpha_{\Xi} \alpha_{\Lambda}+\bar{\alpha}_{\Xi} \bar{\alpha}_{\Lambda}} \approx A_{\Xi}+A_{\Lambda}
$$

By using hyperons produced at $0^{\circ}$ (i.e., aligned with the incoming proton beam), an unpolarized $\Xi$ sample is obtained, so that the polarization of the daughter $\Lambda$ is exactly given by $\alpha_{\Xi}$. The power of this technique derives from the relatively large $|\alpha|$ value for the $\Xi^{-} \rightarrow \Lambda \pi^{-}$ decay $\left(\alpha_{\Xi}=-0.458 \pm 0.012[28]\right)$.

Subsequently to E756, this technique was used in the "HyperCP" experiment (Fermilab E871) [61, 62], which ran during 1996-99 and has published the world's best limits on hyperon $C P$ violation, based so far on about $5 \%$ of the recorded ${ }^{\left(\Xi^{)}\right.} \mp \rightarrow{ }^{(} \bar{\Lambda}^{\prime} \pi^{\mp}$ data sample. (The systematics of the full data sample is still under study.) HyperCP recorded the world's largest samples of hyperon and antihyperon decays, including $2.0 \times 10^{9}$ and $0.46 \times 10^{9} \Xi^{-}$ and $\bar{\Xi}^{+}$events, respectively. When the analysis is complete, these should determine $A_{\Xi \Lambda}$ with a statistical uncertainty

$$
\delta A=\frac{1}{2 \alpha_{\Xi} \alpha_{\Lambda}} \sqrt{\frac{3}{N_{\Xi^{-}}}+\frac{3}{N_{\Xi^{+}}}} \approx 2 \times 10^{-4} .
$$

A preliminary result based on the full analysis of the HyperCP 1999 sample, $A_{\Xi \Lambda}=$ $[-6.0 \pm 2.1$ (stat) \pm 2.1 (syst) $] \times 10^{-4}$, was presented this summer [7] (Table 4). The standard model predicts this asymmetry to be of order $10^{-5}[49,51]$ (see Table 5). Thus the HyperCP full-statistics analysis sees an effect substantially in excess of the standard model prediction. Although only at the $2 \sigma$ level of significance, it is evidence for new sources of $C P$ violation in the baryon sector. (A number of standard model extensions predict effects as large as $\mathcal{O}\left(10^{-3}\right)$ [63]). Such an observation could be of relevance to the mysterious mechanism that gave rise to the cosmic baryon asymmetry.

HyperCP has also set the world's first limit on $C P$ violation in $(\bar{\Omega})$ decay, using a sample of $5.46 \times 10^{6} \Omega^{-} \rightarrow \Lambda K^{-}$events and $1.89 \times 10^{6} \bar{\Omega}^{+} \rightarrow \bar{\Lambda} K^{+}$events [64]. Here, as shown by HyperCP [65, 66], parity is only slightly violated: $\alpha=(1.80 \pm 0.24) \times 10^{-2}[28]$. Hence the measured magnitude and uncertainty of the asymmetry parameter $A_{\Omega \Lambda}$ (inversely proportional to $\alpha$ as in Eq. 2) are rather large: [ $-0.4 \pm 9.1$ (stat) \pm 8.5 (syst)] $\times 10^{-2}$ [64]. This asymmetry is predicted to be $\leq 4 \times 10^{-5}$ in the standard model but can be as large as $8 \times 10^{-3}$ if new physics contributes [53].

\subsubsection{Partial-rate asymmetries}

While $C P T$ symmetry requires the lifetimes of a particle and its antiparticle to be identical, partial-rate asymmetries violate only $C P$. For most hyperon decays, partial-rate asymmetries are expected to be undetectably small [50]. However, this need not be the case for the decays $\Omega^{-} \rightarrow \Lambda K^{-}$and $\Omega^{-} \rightarrow \Xi^{0} \pi^{-}$, for which the particle/antiparticle partial-rate 
Table 4: Summary of experimental limits on $C P$ violation in hyperon decay; the hyperons studied are indicated by ${ }^{*},{ }^{\dagger}$, and ${ }^{\ddagger}$.

\begin{tabular}{|c|c|c|c|c|c|}
\hline Exp’t & Facility & Year & Ref. & Modes & ${ }^{*} A_{\Lambda} /{ }^{\dagger} A_{\Xi \Lambda} /{ }^{\ddagger} A_{\Omega \Lambda}$ \\
\hline R608 & ISR & 1985 & {$[56]$} & $p p \rightarrow \Lambda X, p p \rightarrow \bar{\Lambda} X$ & $-0.02 \pm 0.14^{*}$ \\
\hline DM2 & Orsay & 1988 & {$[57]$} & $e^{+} e^{-} \rightarrow J / \psi \rightarrow \Lambda \bar{\Lambda}$ & $0.01 \pm 0.10^{*}$ \\
\hline PS185 & LEAR & 1997 & {$[58]$} & $\bar{p} p \rightarrow \bar{\Lambda} \Lambda$ & $0.006 \pm 0.015^{*}$ \\
\hline CLEO & CESR & 2000 & {$[60]$} & $\begin{array}{c}e^{+} e^{-} \rightarrow \Xi^{-} X, \Xi^{-} \rightarrow \Lambda \pi^{-}, \\
e^{+} e^{-} \rightarrow \bar{\Xi}^{+} X, \Xi^{+} \rightarrow \bar{\Lambda} \pi^{+}\end{array}$ & $-0.057 \pm 0.064 \pm 0.039^{\dagger}$ \\
\hline E756 & FNAL & 2000 & {$[59]$} & $\begin{array}{c}p N \rightarrow \Xi^{-} X, \Xi^{-} \rightarrow \Lambda \pi^{-} \\
p N \rightarrow \bar{\Xi}^{+} X, \Xi^{+} \rightarrow \bar{\Lambda} \pi^{+}\end{array}$ & $0.012 \pm 0.014^{\dagger}$ \\
\hline HyperCP & FNAL & 2004 & {$[61]$} & $\begin{array}{c}p N \rightarrow \Xi^{-} X, \Xi^{-} \rightarrow \Lambda \pi^{-} \\
p N \rightarrow \bar{\Xi}^{+} X, \bar{\Xi}^{+} \rightarrow \bar{\Lambda} \pi^{+}\end{array}$ & $(0.0 \pm 6.7) \times 10^{-4 \dagger, \S}$ \\
\hline HyperCP & FNAL & 2006 & {$[64]$} & $\begin{array}{l}p N \rightarrow \Omega^{-} X, \Omega^{-} \rightarrow \Lambda K^{-} \\
p N \rightarrow \bar{\Omega}^{+} X, \bar{\Omega}^{+} \rightarrow \bar{\Lambda} K^{+}\end{array}$ & $-0.004 \pm 0.12^{\ddagger}$ \\
\hline HyperCP & FNAL & 2008 & {$[7]$} & $\begin{array}{c}p N \rightarrow \Xi^{-} X, \Xi^{-} \rightarrow \Lambda \pi^{-} \\
p N \rightarrow \bar{\Xi}^{+} X, \Xi^{+} \rightarrow \bar{\Lambda} \pi^{+}\end{array}$ & $(-6.0 \pm 3.0) \times 10^{-4 \dagger, \boldsymbol{\Phi}}$ \\
\hline
\end{tabular}

$\S$ Based on $\approx 5 \%$ of the HyperCP data sample; analysis of the full sample is still in progress.

๑ Preliminary result of full analysis.

Table 5: Summary of predicted hyperon $C P$ asymmetries.

\begin{tabular}{lccccc}
\hline \hline Asymm. & Mode & SM & Ref. & NP & Ref. \\
\hline \hline$A_{\Lambda}$ & $\Lambda \rightarrow p \pi$ & $\lesssim 4 \times 10^{-5}$ & {$[51]$} & $\lesssim 6 \times 10^{-4}$ & {$[67]$} \\
$A_{\Xi \Lambda}$ & $\Xi^{\mp} \rightarrow \Lambda \pi, \Lambda \rightarrow p \pi$ & $\lesssim 5 \times 10^{-5}$ & {$[51]$} & $\leq 1.9 \times 10^{-3}$ & {$[68]$} \\
$A_{\Omega \Lambda}$ & $\Omega \rightarrow \Lambda K, \Lambda \rightarrow p \pi$ & $\leq 4 \times 10^{-5}$ & {$[53]$} & $\leq 8 \times 10^{-3}$ & {$[53]$} \\
$\Delta_{\Xi \pi}$ & $\Omega \rightarrow \Xi^{0} \pi$ & $2 \times 10^{-5}$ & {$[52]$} & $\leq 2 \times 10^{-4 *}$ & {$[52]$} \\
$\Delta_{\Lambda K}$ & $\Omega \rightarrow \Lambda K$ & $\leq 1 \times 10^{-5}$ & {$[53]$} & $\leq 1 \times 10^{-3}$ & {$[53]$} \\
\hline \hline
\end{tabular}

* Once they are taken into account, large final-state interactions may increase this prediction [76]. 
asymmetries could be as large as $2 \times 10^{-5}$ in the standard model and one to two orders of magnitude larger if non-SM contributions are appreciable $[52,53]$. The quantities to be measured are

$$
\begin{aligned}
\Delta_{\Lambda K} & \equiv \frac{\Gamma\left(\Omega^{-} \rightarrow \Lambda K^{-}\right)-\Gamma\left(\bar{\Omega}^{+} \rightarrow \bar{\Lambda} K^{+}\right)}{\Gamma\left(\Omega^{-} \rightarrow \Lambda K^{-}\right)+\Gamma\left(\overline{\Omega^{+}} \rightarrow \bar{\Lambda} K^{+}\right)}, \quad \Delta_{\Xi \pi} \equiv \frac{\Gamma\left(\Omega^{-} \rightarrow \Xi^{0} \pi^{-}\right)-\Gamma\left(\bar{\Omega}^{+} \rightarrow \bar{\Xi}^{0} \pi^{+}\right)}{\Gamma\left(\Omega^{-} \rightarrow \Xi^{0} \pi^{-}\right)+\Gamma\left(\bar{\Omega}^{+} \rightarrow \bar{\Xi}^{0} \pi^{+}\right)} \\
& \approx \frac{1}{2 \Gamma}(\Gamma-\bar{\Gamma})=0.5(1-\Gamma / \bar{\Gamma}) \\
& \approx 0.5(1-N / \bar{N}),
\end{aligned}
$$

where in the last step we have assumed nearly equal numbers $(N)$ of $\Omega$ and $(\bar{N})$ of $\bar{\Omega}$ events, as would be the case in $\bar{p} p$ annihilation. Sensitivity at the $10^{-4}$ level then requires $\mathcal{O}\left(10^{7}\right)$ reconstructed events. Measuring such a small branching-ratio difference reliably will require the clean, exclusive $\bar{\Omega}^{+} \Omega^{-}$event sample produced less than a $\pi^{0}$ mass above threshold, or $4.938<p_{\bar{p}}<5.437 \mathrm{GeV} / c$.

\subsubsection{Hyperon sensitivity estimates}

There have been a number of measurements of hyperon production by low-energy antiprotons. Johansson et al. [69] report cross sections measured by PS185 at LEAR, but the maximum LEAR $\bar{p}$ momentum $(2 \mathrm{GeV} / c)$ was insufficient to produce $\Xi$ 's or $\Omega$ 's. Chien et $a l$. [70] report measurements of a variety of hyperon final states performed with the BNL 80inch liquid-hydrogen bubble chamber in a $6.935 \mathrm{GeV} / c$ electrostatically separated antiproton beam at the AGS; Baltay et al. [71] summarize data taken at lower momenta. In 80,000 pictures Chien et al. observed some 1,868 hyperon or antihyperon events, corresponding to a total hyperon-production cross section of $1.310 \pm 0.105 \mathrm{mb}$ [70]. The corresponding cross section measured at $3.7 \mathrm{GeV} / c$ was $720 \pm 30 \mu \mathrm{b}$, and $438 \pm 52 \mu \mathrm{b}$ at $3.25 \mathrm{GeV} / c$ [71]. The inclusive hyperon-production cross section at $5.4 \mathrm{GeV} / c$ is thus about $1 \mathrm{mb}$. At $2 \times 10^{32} \mathrm{~cm}^{-2} \mathrm{~s}^{-1}$ this amounts to some $2 \times 10^{5}$ hyperon events produced per second, or $2 \times 10^{12}$ per year. (As discussed below, experience suggests that a data-acquisition system that can cope with such a high event rate is both feasible and reasonable in cost.)

To estimate the exclusive $\bar{p} p \rightarrow \bar{\Omega} \Omega$ cross section requires some extrapolation, since it has yet to be measured (moreover, even for $\bar{p} p \rightarrow \bar{\Xi}^{+} \Xi^{-}$only a few events have been seen). A rule of thumb is that each strange quark "costs" between one and two orders of magnitude in cross section, reflecting the effect of the strange-quark mass on the hadronization process. This is borne out by e.g. HyperCP, in which $2.1 \times 10^{9} \Xi^{-} \rightarrow \Lambda \pi^{-}$and $1.5 \times 10^{7} \Omega^{-} \rightarrow \Lambda K^{-}$ decays were reconstructed [62]; given the $160 \mathrm{GeV} / c$ hyperon momentum and $6.3 \mathrm{~m}$ distance from HyperCP target to decay pipe, this corresponds to $\approx 30 \Xi^{-}$'s per $\Omega^{-}$produced at the target. A similar ratio is observed in HERA- $B$ [72]. In exclusive $\bar{p} p \rightarrow \bar{Y} Y$ production (where $Y$ signifies a hyperon) there may be additional effects, since as one proceeds from $\Lambda$ to $\Xi$ to $\Omega$ fewer and fewer valence quarks are in common between the initial and final states. Nevertheless, the cross section for $\bar{\Xi}^{+} \Xi^{-}$somewhat above threshold $\left(p_{\bar{p}} \approx 3.5 \mathrm{GeV} / c\right)$ is $\approx 2 \mu \mathrm{b}[73,71,74]$, or about $1 / 30$ of the corresponding cross section for $\bar{\Lambda} \Lambda$. Thus the $\approx 65 \mu \mathrm{b}$ cross section measured for $\bar{p} p \rightarrow \bar{\Lambda} \Lambda$ at $p_{\bar{p}}=1.642 \mathrm{GeV} / c$ at LEAR [69] implies $\sigma(\bar{p} p \rightarrow \bar{\Omega} \Omega) \sim 60 \mathrm{nb}$ at $5.4 \mathrm{GeV} / c$.

For purposes of discussion we take $60 \mathrm{nb}$ as a plausible estimate of the exclusive production cross section. ${ }^{8}$ At luminosity of $2.0 \times 10^{32} \mathrm{~cm}^{-2} \mathrm{~s}^{-1}$, some $1.2 \times 10^{8} \bar{\Omega} \Omega$ events are

\footnotetext{
${ }^{8}$ This estimate will be testable in the upgraded MIPP experiment [75].
} 
then produced in a nominal 1-year run $\left(1.0 \times 10^{7} \mathrm{~s}\right)$. Assuming acceptance times efficiency of $50 \%$ (possibly an overestimate, but comparable to that for $\chi_{c}$ events in E760), and given the various branching ratios [28], we estimate ${ }^{(} \bar{N}_{\Xi \pi}^{)}=1.4 \times 10^{7}$ events each in $\Omega^{-} \rightarrow \Xi^{0} \pi^{-}$ and $\bar{\Omega}^{+} \rightarrow \bar{\Xi}^{0} \pi^{+}$, and $\left.{ }^{(} \bar{N}_{\Lambda K}\right)=4.1 \times 10^{7}$ events each in $\Omega^{-} \rightarrow \Lambda K^{-}$and $\bar{\Omega}^{+} \rightarrow \bar{\Lambda} K^{+}$, giving the following statistical sensitivities for partial-rate asymmetries:

$$
\begin{aligned}
\delta \Delta_{\Xi \pi} & \approx \frac{0.5}{\sqrt{N_{\Xi \pi}}} \approx 1.3 \times 10^{-4}, \\
\delta \Delta_{\Lambda K} & \approx \frac{0.5}{\sqrt{N_{\Lambda K}}} \approx 7.8 \times 10^{-5} .
\end{aligned}
$$

Tandean and Valencia [52] have estimated $\Delta_{\Xi \pi} \approx 2 \times 10^{-5}$ in the standard model but possibly an order of magnitude larger with new-physics contributions. Tandean [53] has estimated $\Delta_{\Lambda K}$ to be $\leq 1 \times 10^{-5}$ in the standard model but possibly as large as $1 \times 10^{-3}$ if new physics contributes. (The large sensitivity of $\Delta_{\Lambda K}$ to new physics in this analysis arises from chromomagnetic penguin operators and final-state interactions via $\Omega \rightarrow \Xi \pi \rightarrow \Lambda K$ [53]. ${ }^{9}$ The sensitivity in $\Delta_{\Xi \pi}$ should thus be similar to that in $\Delta_{\Lambda K}$.) It is worth noting that these potentially large asymmetries arise from parity-conserving interactions and hence are limited by constraints from $\epsilon_{K}[52,53]$; they are independent of $A_{\Lambda}$ and $A_{\Xi}$, which arise from the interference of parity-violating and parity-conserving processes [76]. Table 5 summarizes predicted hyperon $C P$ asymmetries.

Of course, the experimental sensitivities will include systematic components whose estimation will require careful and detailed simulation studies, beyond the scope of this Letter of Intent. Nevertheless, the potential power of the technique is apparent: the experiment discussed here will represent a substantial improvement over current sensitivity to Omega angular-distribution $C P$ asymmetries, and it may be capable of observing, via partial-rate asymmetries, the effects of new physics in Omega $C P$ violation.

\subsection{Study of FCNC hyperon decays}

In addition to its high-rate charged-particle spectrometer, HyperCP had a muon detection system aimed at studying rare decays of hyperons and charged kaons [62, 77, 8]. Among recent HyperCP results is the observation of the rarest hyperon decay ever seen, $\Sigma^{+} \rightarrow$ $p \mu^{+} \mu^{-}$[8]. Surprisingly, as shown in Figs. 3 and 4, based on the 3 observed events, the decay is consistent with being two-body, i.e., $\Sigma^{+} \rightarrow p X^{0}, X^{0} \rightarrow \mu^{+} \mu^{-}$, with $X^{0}$ mass $m_{X^{0}}=214.3 \pm 0.5 \mathrm{MeV} / c^{2}$. At the current level of statistics this interpretation is of course not definitive: the probability that the 3 signal events are consistent with the standard model form-factor spectrum of Fig. $4 \mathrm{a}$ is estimated at $0.8 \%$. The measured branching ratio is $[3.1 \pm 2.4$ (stat) \pm 1.5 (syst) $] \times 10^{-8}$ assuming the intermediate $\Sigma^{+} \rightarrow p X^{0}$ two-body decay, or $\left[8.6_{-5.4}^{+6.6}\right.$ (stat) \pm 5.5 (syst) $] \times 10^{-8}$ assuming three-body $\Sigma^{+}$decay.

This result is particularly intriguing in view of the proposal by D. S. Gorbunov and co-workers [78] that there should exist in certain nonminimal supersymmetric models a pair of "sgoldstinos" (supersymmetric partners of Goldstone fermions). These can be scalar or pseudoscalar and could be low in mass. A light scalar particle coupling to hadronic matter and to muon pairs at the required level is ruled out by the failure to observe it in kaon decays; however, a pseudoscalar sgoldstino with $\approx 214 \mathrm{MeV} / c^{2}$ mass would be consistent with all

\footnotetext{
${ }^{9}$ Large final-state interactions of this sort should also affect $\Delta_{\Xi \pi}$ but were not included in that prediction $[52,76]$.
} 


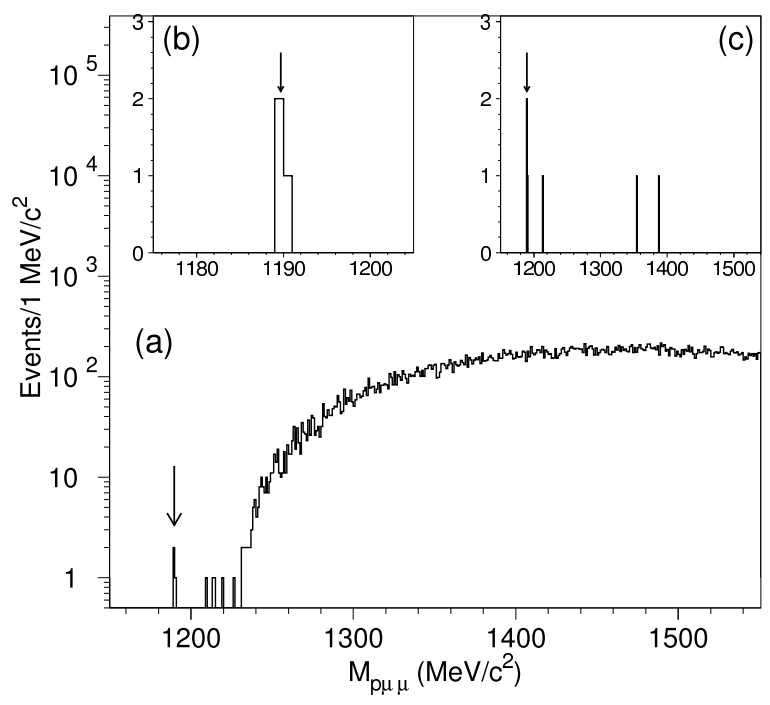

Figure 3: Mass spectrum for 3-track final states consistent with being single-vertex $p \mu^{+} \mu^{-}$ events in HyperCP positive-beam data sample: (a) wide mass range (semilog scale); (b) narrow range around $\Sigma^{+}$mass; (c) after application of additional cuts as described in Ref. [8]. (Arrows indicate mass of $\Sigma^{+}$.)
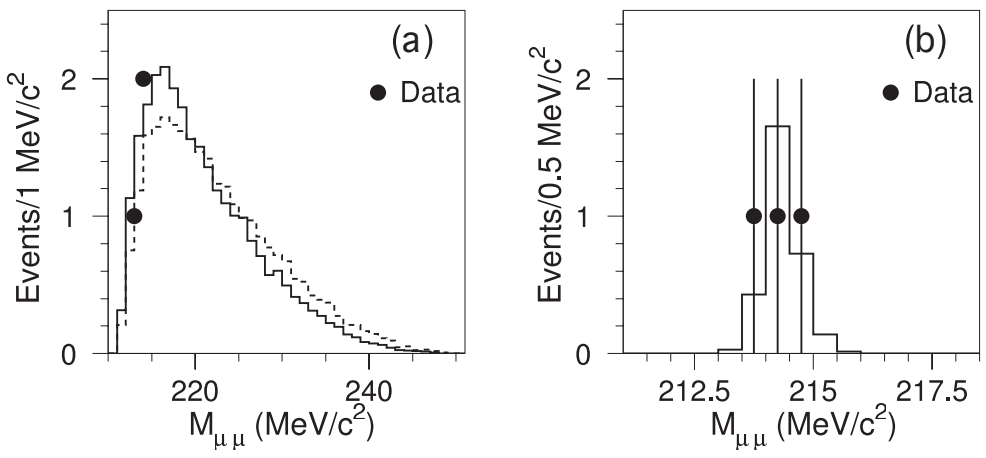

Figure 4: Dimuon mass spectrum of the three HyperCP $\Sigma^{+} \rightarrow p \mu^{+} \mu^{-}$candidate events compared with Monte Carlo spectrum assuming (a) standard model virtual-photon form factor (solid) or isotropic decay (dashed), or (b) decay via a narrow resonance $X^{0}$. 
available data $[79,80,81]$. An alternative possibility has recently been advanced by He, Tandean, and Valencia [82]: the $X^{0}$ could be the light pseudoscalar Higgs boson in the nextto-minimal supersymmetric standard model (the $A_{1}^{0}$ ). Thus, the lightest supersymmetric particle may already have been glimpsed.

While it might be desirable to study $\Sigma^{+}$and $\bar{\Sigma}^{-}$decays using clean, exclusive $\bar{p} p \rightarrow$ $\bar{\Sigma}^{-} \Sigma^{+}$events just above threshold, this would require a $\bar{p}$ momentum (see Table 1 ) well below what has been accomplished in the past by deceleration in the Antiproton Accumulator, as well as very high luminosity to access the $\mathcal{O}\left(10^{-8}\right)$ branching ratio. An experimentally less challenging but equally interesting objective is the corresponding FCNC decay of the $\Omega^{-}$, with predicted branching ratio of order $10^{-6}$ if the $X^{0}$ seen in $\Sigma^{+} \rightarrow p \mu^{+} \mu^{-}$is real [79]. ${ }^{10}$ (The larger predicted branching ratio reflects the additional phase space available compared to that in $\Sigma^{+} \rightarrow p \mu^{+} \mu^{-}$.) As above, assuming $2 \times 10^{32}$ luminosity and $50 \%$ acceptance times efficiency, 120 or 44 events are predicted in the two cases (pseudoscalar or axial-vector $X^{0}$ ) that appear to be viable $[79,80]$ :

$$
\begin{aligned}
\mathcal{B}\left(\Omega^{-} \rightarrow \Xi^{-} X_{P} \rightarrow \Xi^{-} \mu^{+} \mu^{-}\right) & =\left(2.0_{-1.2}^{+1.6} \pm 1.0\right) \times 10^{-6} \\
\mathcal{B}\left(\Omega^{-} \rightarrow \Xi^{-} X_{A} \rightarrow \Xi^{-} \mu^{+} \mu^{-}\right) & =\left(0.73_{-0.45}^{+0.56} \pm 0.35\right) \times 10^{-6}
\end{aligned}
$$

Given the large inclusive hyperon rates at $\sqrt{s} \approx 3.5$ to $4.3 \mathrm{GeV}$, sufficient sensitivity might also be available at those energies to confirm the HyperCP $\Sigma^{+} \rightarrow p \mu^{+} \mu^{-}$results.

\section{Apparatus}

If the cross-section estimates above had measurements to back them up, the potential of this experiment to make world-leading measurements, including the world's most sensitive searches for new physics in the areas described, would be on more solid ground. We would then be designing a new experiment from scratch, the cost of which would clearly be worthwhile. Instead, what we have are plausibility arguments that the world's best measurements of their kind might be possible at the Antiproton Source. Under these circumstances, we believe that an experiment is still worthwhile, but clearly, given the uncertainties on physics reach, in many respects it will be an exploratory effort, and its cost should therefore be kept modest.

Our starting point is the E835 detector (Fig. 5). Many of the components of this detector have been stored intact since E835 was decommissioned, thus they can be reassembled at relatively small effort and cost. This would suffice for many of the charmonium and relatedstate studies discussed above. E760 and E835 relied for triggering on electromagnetic-energy deposition to suppress the high interaction rate $\left(10^{6} \mathrm{~Hz}\right)$ of minimum-bias $\bar{p} p \rightarrow n$ pions events $\left(\langle n\rangle \approx 5,\left\langle n_{c h}\right\rangle \approx 2\right)$, and on Cherenkov detection and electromagnetic calorimetry to suppress backgrounds in offline analysis. While ideal for charmonium studies, this approach is not workable for charm or hyperon triggering and reconstruction.

We therefore propose to replace the E835 inner detectors with a magnetic spectrometer (see Fig. 6). This would be a small, thin superconducting solenoid enclosing scintillating-fiber tracking detectors and silicon vertex detectors (e.g., of the type developed for BTeV [83]). The cost of superconducting magnets is monitored by LBNL's M. Green and reported in periodic papers at the Applied Superconductivity Workshops [85]. The solenoid we consider should cost in the vicinity of $1 \mathrm{M} \$$. We choose scintillating fibers because the

\footnotetext{
${ }^{10}$ The standard-model prediction is $\mathcal{B}\left(\Omega^{-} \rightarrow \Xi^{-} \mu^{+} \mu^{-}\right)=6.6 \times 10^{-8}$ [84].
} 


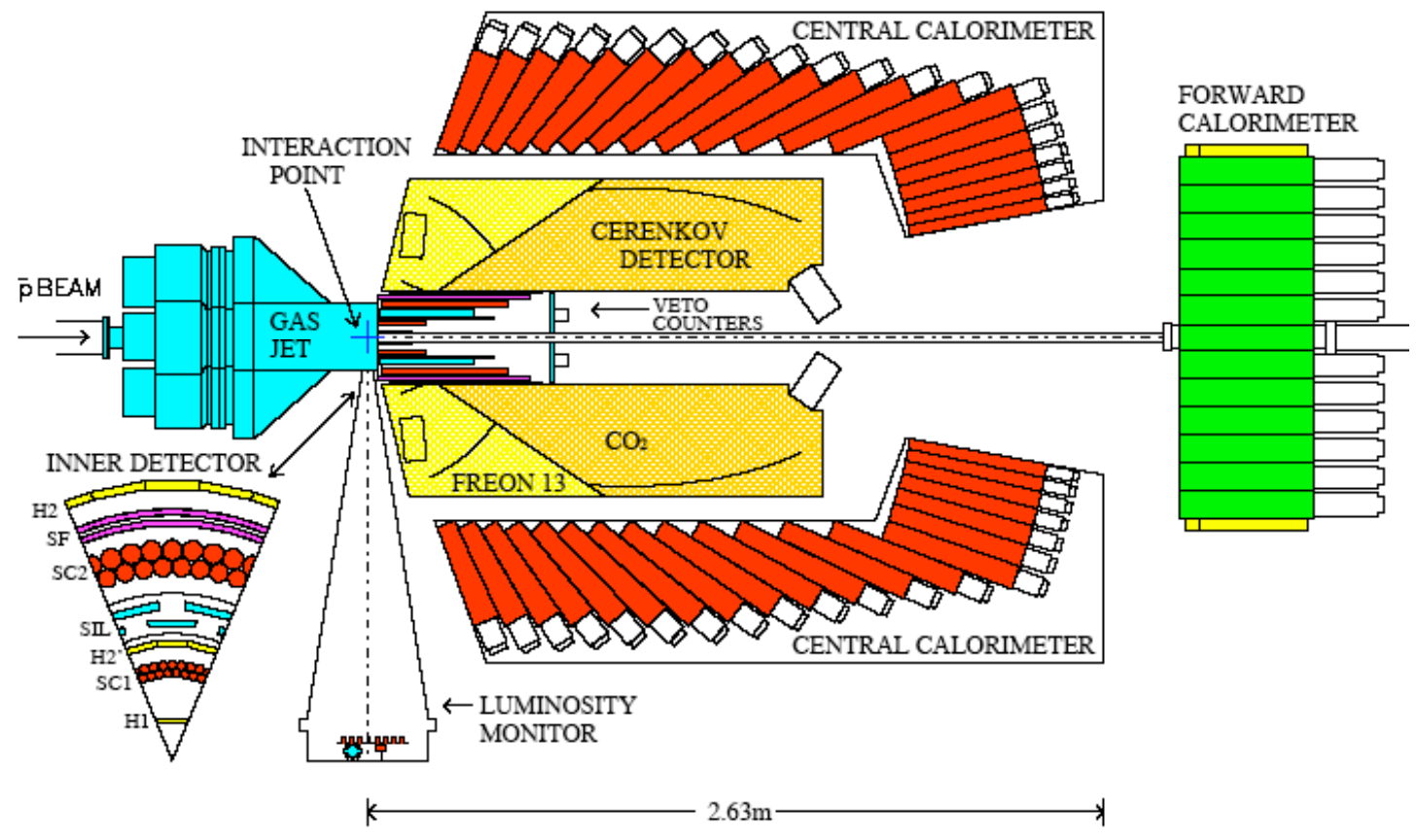

Figure 5: E835 apparatus layout (from [11]).

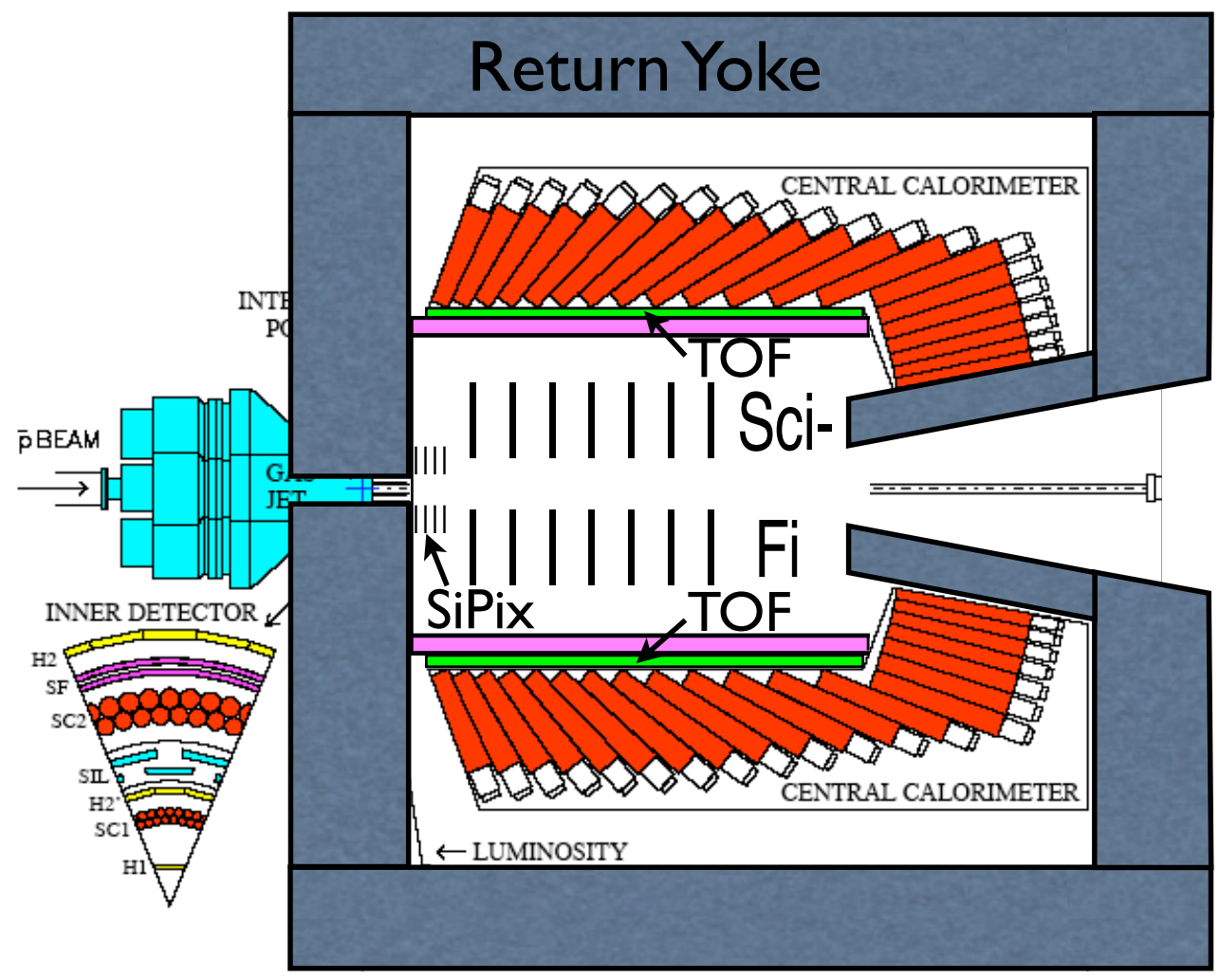

Figure 6: Sketch of upgraded E835 apparatus as discussed in text: $1 \mathrm{~T}$ solenoid shown in magenta, TOF counters in green. Return yoke should be as little iron as necessary. 
fibers themselves are inexpensive, and a very capable readout system for scintillating-fiber detectors should become available from $\mathrm{D} \varnothing[86]$ once the Tevatron finishes. Moving the readout system from $\mathrm{D} \varnothing$ will be far simpler and more cost-effective then building a new one from scratch.

Triggering would be based on track multiplicities at large angles and evidence of separated decay vertices [87]. Compared to $\mathrm{BTeV}$, the $\bar{p} p$ experiment has a low charged-particle rate $\left(\mathrm{a}\right.$ few $\times 10^{7} \mathrm{~Hz}$ ) and a much more localized interaction region. Thus a much more modest and less costly installation than envisioned for BTeV should suffice, along with a reduced version of the $\mathrm{BTeV}$ data-acquisition system. ${ }^{11}$

Hadron identification is highly desirable, e.g., in order to suppress backgrounds to charm decays. In the momentum range of interest, this can be accomplished by time-of-flight measurement. In addition, electrons and muons can be identified from their response in the calorimeter. (Studies are in progress and will be reported as results become available.)

\section{Competition for Resources}

There has been discussion of reusing parts of the Antiproton Source in order to create a proton beam suitable for the mu2e experiment. This reuse has not been studied in detail and is not yet planned, thus may not happen. If it does happen, given the time it will take to fund and implement that experiment, there is ample time - as well as a strong physics case - for a few years of antiproton running before it will be ready. Even if such reuse of the Antiproton Source is undertaken, in the longer term, the Project X beam will be too intense to buffer in the Antiproton Source, requiring a new, larger-acceptance and better-shielded $8 \mathrm{GeV}$ ring to be built, and once again freeing the Antiproton Source to do what it does best.

The Fermilab Director has expressed the view that the mounting of this experiment cannot be undertaken by Fermilab as the lab's staff is already stretched too thinly. We are actively seeking new collaborators in the US and Europe who can take on part of this burden. With some engineering and technical assistance now, we will be able to identify those technical solutions that will minimize the needed time, cost, and effort.

\section{Our Request}

We request from Fermilab the modest support needed to study the proposed experiment in greater detail and develop a proposal. This will require of order a physicist-FTE plus technical support to develop a cost estimate and an implementation plan.

\section{Summary and Conclusions}

We are proposing the world's best experiment on charm mixing and $C P$ violation, hyperon $C P$ violation and rare decays, and charmonium and related states. Because of existing equipment from previous experiments, it can be assembled quickly and at modest cost. In the face of current budget exigencies, this is a practical way to keep Fermilab at the

\footnotetext{
${ }^{11}$ Like the experiment we consider here, $\mathrm{BTeV}$ was designed to operate at a luminosity of $2 \times 10^{32} \mathrm{~cm}^{-2} \mathrm{~s}^{-1}$. The cross section at $\sqrt{s}=3.5 \mathrm{GeV}$ is only $\approx 20 \%$ less than that at $2 \mathrm{TeV}$, but the mean charged multiplicity is smaller by a factor $\approx 20[28]$.
} 
forefront of flavor physics. The experiment exploits Fermilab's unique capability to provide an intense beam of medium-energy antiprotons, and it offers unique discovery potential. 


\section{References}

[1] T. Eriksson, in Proc. LEAP05 Conf., AIP Conf Proc. 796, 389 (2005).

[2] See http://www.gsi.de/fair/index_e.html

[3] See http://www.slac.stanford.edu/xorg/hfag/charm/ICHEP08/results_mix+cpv.html and E. Barberio et al., arXiv:0808.1297 [hep-ex].

[4] See e.g. I. I. Bigi and N. Uraltsev, Nucl. Phys. B 592 (2001) 92; A. A. Petrov, arXiv:hepph/0311371; Nucl. Phys. Proc. Suppl. 142 (2005) 333.

[5] See e.g. Sec. 3.9 of G Buchalla et al., Report of Working Group 2 of the CERN Workshop "Flavour in the era of the LHC", Geneva, Switzerland, November 2005-March 2007, arXiv:0801.1833 [hep-ph] (2008).

[6] E. Eichten. K, Lane, and C. Quigg, Phys. Rev. D 73, 014014 (2006); Erratum-ibid. D 73, 079903 (2006).

[7] C. Materniak, presented at The Eighth International Conference on Hyperons, Charm and Beauty Hadrons (BEACH08), Columbia, SC 22-28 June 2008, available at http://beach2008.sc.edu/includes/documents/sessions/materniak.talk.pdf

[8] H. K. Park et al. [HyperCP Collaboration], Phys. Rev. Lett. 94, 021801 (2005).

[9] G. Garzoglio et al. [E835 Collaboration], Nucl. Instrum. Meth. A 519, 558 (2004).

[10] T. A. Armstrong et al. [E835 Collaboration], Phys. Rev. D 47, 772 (1993).

[11] See http://www.e835.to.infn.it/

[12] M. Kotulla et al. [Panda Collaboration], Technical Progress Report for: Panda, U. Wiedner, Spokesperson, available from http://www-panda.gsi.de/archive/public/panda_tpr.pdf

[13] K. Ehret, Nucl. Instr. Meth. A 446, (2000) 190.

[14] E. Golowich, J. Hewett, S. Pakvasa, A. A. Petrov, Phys. Rev. D 76, 095009 (2007).

[15] See e.g. Y. Grossman, A. L. Kagan, Y. Nir, Phys. Rev. D 75, 036008 (2007).

[16] See e.g. J. L. Hewett, "Searching for New Physics with Charm," Proc. LISHEP95 Workshop, Rio de Janeiro, Brazil, Feb. 20-22, 1995, p. 171.

[17] S. Pakvasa, "Charm as Probe of New Physics," in The Future of High-Sensitivity Charm Experiments, D. M. Kaplan and S. Kwan, eds., FERMILAB-Conf-94/190 (1994), p. 85.

[18] M. D. Sokoloff and D. M. Kaplan, "Physics of an Ultrahigh-Statistics Charm Experiment," in Heavy Quarks at Fixed Target, B. Cox, ed., Frascati Physics Series no. 3 (1994), p. 411.

[19] See e.g. J. L. Rosner, "CP Violation: Past, Present, and Future," hep-ph/0101033, Braz. J. Phys. 31 (2001) 147. 
[20] B. Aubert et al. (BABAR Collaboration), Phys. Rev. Lett. 87, 091801 (2001); K. Abe et al. (Belle Collaboration), Phys. Rev. Lett. 87, 091802 (2001).

[21] M. Golden and B. Grinstein, Phys. Lett. B 222, 501 (1989); F. Buccella et al., Phys. Lett. B 302, 319 (1993) and Phys. Rev. D 51, 3478 (1995); A. Pugliese and P. Santorelli, "Two Body Decays of $D$ Mesons and $C P$ Violating Asymmetries in Charged $D$ Meson Decays," Proc. Third Workshop on the Tau/Charm Factory, Marbella, Spain, 1-6 June 1993, Edition Frontieres (1994), p. 387; G. Burdman, "Charm Mixing and CP Violation in the Standard Model," in The Future of High-Sensitivity Charm Experiments, D. M. Kaplan and S. Kwan, eds., FERMILAB-Conf-94/190 (1994), p. 75.

[22] E. Braaten, private communication.

[23] E. Braaten, Phys. Rev. D 77, 034019 (2008).

[24] Z. Xing, Phys. Lett. B 353 (1995) 313.

[25] S. N. Ganguli et al., Nucl. Phys. B 183, 295 (1981).

[26] M. J. Leitch et al., Phys. Rev. Lett. 72, 2542 (1994).

[27] See e.g. A. A. Petrov, arXiv:0806.2498v1 [hep-ph], and references therein.

[28] C. Amsler et al. [Particle Data Group], Phys. Lett. B 667, 1 (2008).

[29] S. K. Choi et al. [Belle Collaboration], Phys. Rev. Lett. 91, 262001 (2003).

[30] D. Acosta et al. [CDF II Collaboration], Phys. Rev. Lett. 93, 072001 (2004).

[31] V. M. Abazov et al. [DØ Collaboration], Phys. Rev. Lett. 93, 162002 (2004).

[32] B. Aubert et al. [BABAR Collaboration], Phys. Rev. D 71, 071103 (2005).

[33] K. Abe et al., "Evidence for $X(3872) \rightarrow \gamma J / \psi$ and the sub-threshold decay $X(3872) \rightarrow$ $\omega J / \psi, "$ arXiv:hep-ex/0505037.

[34] B. Aubert et al. [BABAR Collaboration], arXiv:0809.0042v1 [hep-ex].

[35] K. Abe et al. [Belle Collaboration], arXiv: hep-ex/0408116.

[36] G. Gokhroo et al. [Belle Collaboration], Phys. Rev. Lett. 97, 162002 (2006).

[37] N. Brambilla et al. [Quarkonium Working Group], Heavy Quarkonium Physics, CERN Yellow Report CERN-2005-005 (2005).

[38] K. Abe et al., "Experimental constraints on the possible $J^{P C}$ quantum numbers of the $X(3872)$," contributed to 22nd International Symposium on Lepton-Photon Interactions at High Energy (LP 2005), Uppsala, Sweden, 30 June - 5 July 2005, arXiv:hepex/0505038.

[39] E. Braaten, "Review of the $X(3872)$," presented at the Int. Workshop on Heavy Quarkonium - 2006, Brookhaven National Laboratory, June 27-30, 2006; available from

http://www.qwg.to.infn.it/WS-jun06/WS4talks/Tuesday_AM/Braaten.pdf 
[40] D. V. Bugg, Phys. Lett. B 598, 8 (2004); Phys. Rev. D 71, 016006 (2005).

[41] L. Maiani, F. Piccinini, A. D. Polosa, and V. Riquer, Phys. Rev. D 71, 014028 (2005); L. Maiani, V. Riquer, F. Piccinini and A. D. Polosa, Phys. Rev. D 72, 031502 (2005); H. Hogaasen, J. M. Richard and P. Sorba, Phys. Rev. D 73, 54013 (2006).

[42] N. A. Tørnqvist, Phys. Lett. B 590, 209 (2004).

[43] T. Barnes, S. Godfrey, Phys. Rev. D 69, 054008 (2004).

[44] E. Braaten, Phys. Rev. D 73, 011501(R) (2006).

[45] T. A. Armstrong et al. [E760 Collaboration], Nucl. Phys. B 373, 35 (1992).

[46] B. Aubert et al. [BABAR Collaboration], Phys. Rev. Lett. 96, 052002 (2006).

[47] See http://belle.kek.jp/superb/.

[48] A. Pais, Phys. Rev. Lett. 3, 242 (1959); O. E. Overseth and S. Pakvasa, Phys. Rev. 184, 1663 (1969); J. F. Donoghue and S. Pakvasa, Phys. Rev. Lett. 55, 162 (1985).

[49] J. F. Donoghue, X.-G. He, S. Pakvasa, Phys. Rev. D 34, 833 (1986); X.-G. He, H. Steger, G. Valencia, Phys. Lett. B 272, 411 (1991).

[50] G. Valencia, "Hyperon CP Violation," in Proc. $\bar{p} 2000$ Workshop, D. M. Kaplan and H. A. Rubin, eds., Illinois Institute of Technology, Chicago, IL 60616, USA, Aug. 3-5, 2000; available from

http://www. capp.iit.edu/ capp/workshops/pbar2000/pbar2000_program.html

[51] J. Tandean, G. Valencia, Phys. Rev. D 67, 056001 (2003).

[52] J. Tandean, G. Valencia, Phys. Lett. B 451, 382 (1999).

[53] J. Tandean, Phys. Rev. D 70, 076005 (2004).

[54] T. D. Lee and C. N. Yang, Phys. Rev. 105, 1761 (1957).

[55] J. F. Donoghue, B. R. Holstein, and G. Valencia, Phys. Lett. 178B, 319 (1986) and Int. J. Mod. Phys. A 2, 319 (1987).

[56] P. Chauvat et al., Phys. Lett. 163B, 273 (1985).

[57] M. H. Tixier et al., Phys. Lett. B 212, 523 (1988).

[58] P. D. Barnes et al., Nucl. Phys. B (Proc. Suppl.) 56A, 46 (1997).

[59] K. B. Luk et al., Phys. Rev. Lett. 85, 4860 (2000).

[60] D. E. Jaffe et al., "Search for Direct CP Violation in $\Xi$ Hyperon Decay," CLNS 98/1587, CLEO 98-16 (2000) (unpublished).

[61] T. Holmstrom et al. [HyperCP Collaboration], Phys. Rev. Lett. 93, 262001 (2004); this analysis represents about $5 \%$ of the total HyperCP data sample.

[62] R. A. Burnstein et al., Nucl. Instrum. Meth. A 541, 516 (2005). 
[63] X.-G. He et al., Phys. Rev. D 61, 071701 (2000).

[64] L. C. Lu et al. [HyperCP Collaboration], Phys. Rev. Lett. 96, 242001 (2006).

[65] Y. C. Chen et al. [HyperCP Collaboration], Phys. Rev. D 71, 051102 (2005).

[66] L. Lu et al. [HyperCP Collaboration], Phys. Lett. B 617, 11 (2005).

[67] D. Chang, X.-G. He, and S. Pakvasa, Phys. Rev. Lett. 74, 3927 (1995).

[68] X.-G. He, H. Murayama, S. Pakvasa, G. Valencia, Phys. Rev. D 61, 071701(R) (2000).

[69] T. Johansson, in Proc. Eighth Int. Conf. on Low Energy Antiproton Physics (LEAP '05), Bonn, Germany, 16-22 May 2005, AIP Conf. Proc. 796, 95 (2005).

[70] C. Y. Chien et al., Phys. Rev. 152, 1181 (1966).

[71] C. Baltay et al., Phys. Rev. 140, B1027 (1965).

[72] Markward Britsch, "Hyperon Production in Proton-Nucleus Collisions at 42-GeV Center of Mass Energy," diploma thesis, Max-Planck-Institute for Nuclear Physics, Heidelberg (2003).

[73] N. Hamann et al., report CERN/SPSLC 92019, SPSLC/M491, 30 March 1992.

[74] High-Energy Reactions Analysis Group, report CERN-HERA-84-01 (1984).

[75] D. Isenhower et al., Fermilab Proposal 960, available from http://ppd.fnal.gov/experiments/e907/notes/MIPPnotes/public/pdf/ MIPP0138/MIPP0138.pdf

[76] J. Tandean, private communication.

[77] H. K. Park et al. [HyperCP Collaboration], Phys. Rev. Lett. 88, 111801 (2002).

[78] D. S. Gorbunov and V. A. Rubakov, Phys. Rev. D 64, 054008 (2001); D. S. Gorbunov, Nucl. Phys. B 602, 213 (2001).

[79] X.-G. He, J. Tandean, G. Valencia, Phys. Lett. B 631, 100 (2005).

[80] N. G. Deshpande, G. Eilam, J. Jiang, Phys. Lett. B 632, 212 (2006).

[81] C. Q. Geng and Y. K. Hsiao, Phys. Lett. B 632, 215 (2006);

D. S. Gorbunov and V. A. Rubakov, Phys. Rev. D 73, 035002 (2006).

[82] X.-G. He, J. Tandean, G. Valencia, Phys. Rev. Lett. 98, 081802 (2007).

[83] M. A. Turqueti et al., "Pixel Multichip Module Development at Fermilab," in Proc. 11th Workshop on Electronics for LHC and Future Experiments, CERN-2005-011, CERN-LHCC-2005-028, p. 205, available from http://indico.cern.ch/materialDisplay . py?contribId=73\&amp; sessionId=

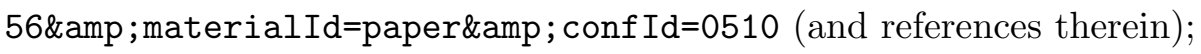
L. Uplegger et al., "First Look at the Beam Test Results of the FPIX2 Readout Chip for the BTeV Silicon Pixel Detector," IEEE Trans. Nucl. Sci. 53, 409 (2006). 
[84] R. Safadi and P. Singer, Phys. Rev. D 37, 697 (1988) [erratum ibid. D 42, 1856 (1990)].

[85] See e.g. M. A. Green and B. P. Strauss, "The Cost of Superconducting Magnets as a Function of Stored Energy and Design Magnetic Induction times the Field Volume," in IEEE Transactions on Applied Superconductivity 18, No. 2, LBNL-63482 (2007).

[86] V. M. Abazov et al., Nucl. Instrum. Meth. A 565 (2006) 463.

[87] E. E. Gottschalk (for the BTeV collaboration), in Proc. 10th Int. Conf. on B Physics at Hadron Machines (BEAUTY 2005), Assisi, Perugia, Italy, 20-24 June 2005, Nucl. Phys. Proc. Suppl. 156, 252 (2006) (and references therein). 